\title{
Existe a Constipação após Histerectomia? Avaliação Clínica e Manométrica
}

\author{
Is There Constipation After Hysterectomy? Clinical and Manometric Evaluation
}

\author{
${ }^{1}$ MARIAAUXILIADORAPROLUNGATTI CESAR; ${ }^{2}$ LILIAN BORGHETTIANTUNES; \\ ${ }^{3}$ RENATAMARTINUZZO DEAGUIAR
}

\begin{abstract}
1. Mestre e Doutora em medicina pela Faculdade de Ciências Médicas da Santa Casa de São Paulo. Professor Assistente Doutor da disciplina de Cirurgia do Departamento de Medicina da Universidade de Taubaté e Diretora Técnica do Hospital Universitário de Taubaté; ${ }^{2}$ Médica, ex aluna do departamento de medicina da universidade de taubaté;

3. Médica, ex aluna do departamento de medicina da universidade de taubaté .
\end{abstract}

\begin{abstract}
CESAR MAP; ANTUNES LB; AGUIAR RM. Existe a Constipação após Histerectomia? Avaliação Clínica e Manométrica. Rev bras Coloproct, 2010;30(2): 191-198.

RESUMO: A histerectomia é procedimento cirúrgico ginecológico de grande porte mais comum, sendo a maioria das indicações por doença benigna. Dentre as complicações desse procedimento cirúrgico estão a constipação, dispareunia, incontinência para gases e fezes, urgência evacuatória, escapes fecais e distensão abdominal. A constipação tem sido encontrada freqüentemente após a histerectomia principalmente em trabalhos retrospectivos. Objetivo: estudar a incidência de constipação após a histerectomia e as alterações manométricas a ela relacionadas. Métodos: Estudo prospectivo em nove pacientes submetidas à histerectomia total abdominal por mioma. Foi realizado estudo manométrico e aplicação do escore de constipação adotado pela sociedade brasileira de motilidade digestiva no pré e sessenta dias de pós-operatório. Resultados: Os autores encontraram a presença de evacuação incompleta que não ocorria no pós-operatório. Não foram encontradas alterações manométricas e duas pacientes apresentaram dor abdominal à distensão da ampola retal na aferição do volume máximo tolerável. Conclusões: Os achados deste estudo sugerem que alterações intestinais ocorrem após a histerectomia e são principalmente relacionadas ao reto.
\end{abstract}

Descritores: Histerectomia, constipação intestinal, técnicas de diagnóstico e procedimentos, procedimentos cirúrgicos operatórios, complicações pós-operatórias.

\section{INTRODUÇÃO}

A histerectomia é o procedimento cirúrgico ginecológico de grande porte mais comum em ginecologia, sendo a maioria das indicações por doença benigna. ${ }^{(1-3)}$

Dentre as complicações após este procedimento encontram-se a dispareunia ${ }^{(4)}$, infecções, hemorragias, incontinência para gases e fezes, urgência evacuatória, escapes fecais ${ }^{(2-4)}$, inabilidade para percepção de gases e fezes ${ }^{(2)}$, distensão abdominal ${ }^{(4)}$ e constipação. ${ }^{(1,5-9)}$

A constipação após histerectomia vem chamando a atenção, pois, em diferentes serviços, tem sido encontrada uma média de $37 \%$ de constipação após histerectomia, não importando a técnica cirúrgica utilizada.

A constipação é uma das principais complicações e é definida como menos que três evacuações por semana ou a evacuação incompleta, com dificuldade e/ ou esforço por pelo menos $25 \%$ do tempo; (1-5,9-18) e tem sido encontrada freqüentemente após a histerectomia. ${ }^{(1-9)}$ Vários estudos estão em desenvolvimento a fim de estabelecer de estabelecer a relação causal da constipação, através de exames de fisiologia anal. ${ }^{(1-9)}$

A maioria dos autores sugere que alterações intestinais após a histerectomia estariam relacionadas 
a lesões na inervação parassimpática pélvica decorrente da manipulação cirúrgica. ${ }^{(1,5,7,8)}$ Outra associação frequentemente citada seria relacionada à redução dos níveis de prostaglandina, decorrente da retirada do útero uma vez que a presença desse hormônio está relacionada à aceleração da motilidade intestinal. ${ }^{(1,5)}$

Em estudo retrospectivo realizado sobre o hábito intestinal após histerectomia foi encontrado que as pacientes que realizaram histerectomia se consideravam mais constipadas do que os controles, não ocorrendo diferença quanto ao uso de laxantes. ${ }^{(7)} \mathrm{Em}$ outro estudo retrospectivo através da aplicação de questionários em 513 pacientes submetidas à histerectomia abdominal e vaginal foi encontrada constipação em $41 \%$ das pacientes não existindo relação com o tipo de histerectomia realizada. ${ }^{(1)}$

No entanto, os trabalhos prospectivos não demonstram, em sua maioria, a relação da constipação com a histerectomia. (2,4,5,9)

Trabalho realizado através da aplicação de questionário 12 meses antes da cirurgia e seis semanas e seis meses após, encontrou a constipação associada à Síndrome do Intestino Irritável como sintoma predominante no pós-operatório e não a constipação como sintoma isolado. ${ }^{(5)}$

Outro estudo prospectivo avaliou 120 pacientes no pré e pós-operatório através de questionário contendo perguntas sobre dificuldade de esvaziamento evacuatório, evacuação incompleta, digitalização anal, incapacidade para eliminar gases, escape fecal e inabilidade para distinguir gases e fezes. Avaliou o hábito intestinal seis e 12 meses após a histerectomia abdominal e vaginal e não encontrou alteração significativa para sintomas de constipação em ambos os procedimentos. ${ }^{(2)}$

Em concordância com esse estudo, outro avaliou 413 pacientes de modo prospectivo, analisou o hábito intestinal através de entrevista e questionário no pré e pós-operatório. Encontrou que apenas 2\% dos pacientes desenvolveram constipação, porem não definiu o tipo de histerectomia. ${ }^{(9)}$

Através de manometria anorretal, no pré e pósoperatória (três e 11-18 meses após histerectomia) outro estudo analisou 42 pacientes e encontrou que o volume para desencadear o reflexo inibitório anal foi significativamente maior após a histerectomia. Não foram encontradas diferenças entre as pressões de repouso e contração no pré e pós-operatório. ${ }^{(4)}$
A manometria anal é exame muito utilizado na área de coloproctologia para a melhor avaliação de pacientes com constipação intestinal, incontinência fecal e mais recentemente, para avaliação pré - operatória de pacientes que irão se submeter à cirurgia orificial e pélvica ${ }^{(10-18)}$. Diversas pesquisas têm sido realizadas no intuito de verificar o que ocorre relacionado aos esfíncteres anais e ampola retal levando à defecação obstruída utilizando a manometria anal. ${ }^{(11-18)}$

O objetivo desta pesquisa foi verificar a incidência de constipação após a histerectomia e as alterações manométricas a ela relacionadas.

\section{MÉTODO}

Foi realizado um estudo prospectivo, com o objetivo de avaliar a incidência de constipação após histerectomia abdominal e sua relação com alterações manométricas anais.

As pacientes participantes da pesquisa foram pacientes da enfermaria de ginecologia e que se submeteram ao tratamento cirúrgico de mioma através da histerectomia abdominal total no período de julho de 2008 a junho de 2009.

Foram excluídas da amostra aquelas que apresentaram um dos critérios abaixo:

1. Cirurgias prévias anorretais; $\cdot$

2. Constipação prévia à cirurgia (menos que três evacuações por semana ou a evacuação incompleta e com dificuldade)

3. Incontinência fecal prévia à cirurgia

Todas as pacientes assinaram um termo de concordância com o tratamento proposto e ao exame e a pesquisa foi aprovada pela CEP-UNITAU.

Para realização do estudo, as pacientes responderam ao escore de constipação adotado pela sociedade brasileira de Motilidade Digestiva e se submeteram à realização da manometria anal em dois momentos :antes da cirurgia e 60 dias de pós-operatório.

O estudo manométrico foi realizado com o aparelho Alacer acoplado a um sistema, que consiste de um cateter flexível de cinco mm de diâmetro, com oito orifícios radiados por onde ocorre a perfusão de água com velocidade de $18 \mathrm{ml} /$ minuto e, na extremidade distal da sonda , um dedo de luva médio acoplado para insuflar o balão.

Para a realização do exame a paciente permanecia em decúbito lateral esquerdo com as pernas fletidas sobre as coxas. $\mathrm{O}$ cateter era lubrificado em 
solução inerte e introduzido delicadamente no ânus da paciente até ao ponto correspondente a cinco centímetros da borda anal, sendo então retirado a cada centímetro e registrando-se as seguintes medidas:

Pressão Máxima de Repouso (PR): é a pressão obtida com o paciente em situação de relaxamento e mede a atividade do esfincter interno do ânus

Pressão Máxima de Contração (PC): é a pressão obtida com o paciente em situação de contração e mede a atividade do esfincter externo do ânus e músculo pubo-retal

Pressão Mínima de Evacuação (PEv): é a pressão obtida com o paciente em situação de esforço evacuatório e mede a atividade do esfincter interno do ânus

Ponto de Maior Pressão - é o ponto do canal anal em que a pressão de repouso apresenta o maior valor

Comprimento Manométrico do Canal Anal - valor em centímetros do canal anal funcional, obtido através das pressões de repouso.

Sensibilidade retal - obtida através da insuflação do balão (dedo de luva) que se encontra na extremidade do cateter de manometria
o Primeira sensação
o Volume de sensação constante
o Volume máximo tolerável
o Sensação abdominal do conteúdo fecal

A análise estatística foi realizada, com intervalo de confiança de $95 \%$ e o teste estatístico foi o não paramétrico de Mann Whitney.

\section{RESULTADOS}

Foram estudadas nove pacientes, cuja média das idades foi de 46,6 \pm 9,6 anos, variando entre 34 a 64 anos. As pacientes analisadas não referiram constipação no pré-operatório sendo o escore máximo no pré-operatório de cinco e no pós-operatório de onze.

As médias, medianas, valor mínimo, valor máximo e os valores estatísticos pelo método Mann Whitney das pressões de repouso, contração e evacuação aferidas no pré e pós-operatório encontram-se dispostas nas tabelas de 1-3, e não apresentam diferenças estatisticamente significantes.

Em relação ao índice de relaxamento na medida do reflexo inibitório anal, também não observamos diferenças estatisticamente significativas com os diferentes valores de aferição (tabela 4).

Em relação à contração mantida também não foram observadas diferenças significativas entre os grupos nas aferições realizadas segundo a altura do canal anal (tabela 5).

No estudo dos sintomas baseados no de constipação encontramos diferenças entre os dois grupos, quando comparados em relação á sensação de evacuação incompleta que foi maior (tabela 6).

Tabela 1 - Pressões de repouso, segundo a altura do canal anal, de pacientes submetidos a histerectomia.

\begin{tabular}{|c|c|c|c|c|c|c|c|c|}
\hline $\begin{array}{l}\text { Altura } \\
\text { do canal } \\
\text { anal }\end{array}$ & Grupo & $\mathbf{N}$ & Valores & DP & Mínima & Mediana & Máxima & $\begin{array}{c}\text { Análise } \\
\text { estatística } \\
\text { M-W }\end{array}$ \\
\hline \multirow[t]{2}{*}{$1 \mathrm{~cm}$} & 1 & 9 & 56,6 & 34,0 & 22,6 & 52,0 & 110,6 & 0,3 \\
\hline & 2 & 9 & 39,0 & 25,7 & 13,3 & 37,6 & 101,1 & \\
\hline \multirow[t]{2}{*}{$2 \mathrm{~cm}$} & 1 & 9 & 46,9 & 25,1 & 13,2 & 35,5 & 86,1 & 0,4 \\
\hline & 2 & 9 & 35,3 & 16,7 & 15,3 & 38,4 & 67,4 & \\
\hline \multirow[t]{2}{*}{$3 \mathrm{~cm}$} & 1 & 9 & 25,4 & 7,2 & 13,1 & 25,3 & 34,8 & 0,8 \\
\hline & 2 & 9 & 25,4 & 15,1 & 10,1 & 22,8 & 53,5 & \\
\hline \multirow[t]{2}{*}{$4 \mathrm{~cm}$} & 1 & 9 & 13,9 & 8,3 & 2,4 & 13,4 & 28,6 & 0,4 \\
\hline & 2 & 9 & 23,1 & 18,1 & 2,1 & 20,4 & 55,9 & \\
\hline \multirow[t]{2}{*}{$5 \mathrm{~cm}$} & 1 & 9 & 12,6 & 7,2 & 0,8 & 13,1 & 24,6 & 0,09 \\
\hline & 2 & 9 & 20,5 & 9,2 & 11,7 & 19,3 & 41,1 & \\
\hline
\end{tabular}

Grupo 1: pré operatório; Grupo 2: pós-operatório; N: número de pacientes analisados; DP: Desvio padrão. M-W: Mann-Whitney. 
Tabela 2 - Pressões de contração, segundo a altura do canal anal, de pacientes submetidas a histerectomia.

\begin{tabular}{|c|c|c|c|c|c|c|c|c|}
\hline $\begin{array}{l}\text { Altura } \\
\text { do canal } \\
\text { anal }\end{array}$ & Grupo & $\mathbf{N}$ & Média & DP & Mínima & Mediana & Máxima & $\begin{array}{c}\text { Análise } \\
\text { estatística } \\
\text { M-W }\end{array}$ \\
\hline \multirow[t]{2}{*}{$1 \mathrm{~cm}$} & 1 & 9 & 109,5 & 53,3 & 32,3 & 128,4 & 192,5 & 0,4 \\
\hline & 2 & 9 & 92,5 & 61,8 & 18,1 & 89,8 & 215,2 & \\
\hline \multirow[t]{2}{*}{$2 \mathrm{~cm}$} & 1 & 9 & 98,3 & 57,7 & 25,8 & 94,8 & 219,1 & 0,07 \\
\hline & 2 & 9 & 72,1 & 62,0 & 16,5 & 72,2 & 222,6 & \\
\hline \multirow[t]{2}{*}{$3 \mathrm{~cm}$} & 1 & 9 & 60,9 & 24,7 & 16,3 & 57,7 & 108,3 & 0,3 \\
\hline & 2 & 9 & 54,6 & 35,4 & 9,6 & 52,5 & 112,4 & \\
\hline \multirow[t]{2}{*}{$4 \mathrm{~cm}$} & 1 & 9 & 45,7 & 30,3 & 11,8 & 32,6 & 105,1 & 0,6 \\
\hline & 2 & 9 & 51,9 & 29,4 & 12,1 & 51,5 & 97,8 & \\
\hline \multirow[t]{2}{*}{$5 \mathrm{~cm}$} & 1 & 9 & 27,0 & 21,0 & 2,5 & 20,5 & 53,8 & 0,5 \\
\hline & 2 & 9 & 32,9 & 22,3 & 3,3 & 37,8 & 74,6 & \\
\hline
\end{tabular}

Grupo 1: pré operatório; Grupo 2: pós-operatório; $\mathbf{N}$ : número de pacientes analisados; DP: Desvio padrão. M-W: Mann-Whitney.

\section{DISCUSSÃO}

A constipação intestinal é doença muito frequente nos consultórios e ambulatórios de coloproctologia e se sabe que existem uma série de fatores anatômicos e funcionais envolvidos, o que gera uma multiplicidade de pesquisas relacionadas ao diagnóstico e tratamento de forma multifatorial $^{(11-18)}$.

Dentre os tipos de constipação encontramos o trânsito colônico lento em que existe uma lentidão do intestino e a defecação obstruída, em que o intestino funciona com uma velocidade dentro da normalidade, mas a dificuldade é na saída, seja por motivos anatômicos (retocele, sigmoidocele, intussuscepção interna) ou por motivos funcionais como a contração paradoxal do puborretal ou anismus ${ }^{(18)}$.

Dentre os sintomas na defecação obstruída encontramos sensação de rolha, maior tempo evacuatório, necessidade de digitalização anal e sensação de evacuação incompleta, principalmente quando associado ao anismus ${ }^{(18)}$.

Tabela 3 - Pressões de evacuação, segundo a altura do canal anal, de pacientes submetidas a histerectomia.

\begin{tabular}{|c|c|c|c|c|c|c|c|c|}
\hline $\begin{array}{l}\text { Altura } \\
\text { do canal } \\
\text { anal }\end{array}$ & Grupo & $\mathbf{N}$ & Média & DP & Mínima & Mediana & Máxima & $\begin{array}{c}\text { Análise } \\
\text { estatística } \\
\text { M-W }\end{array}$ \\
\hline \multirow{2}{*}{$1 \mathrm{~cm}$} & 1 & 9 & 57,0 & 31,1 & 16,8 & 53,4 & 118,9 & 0,3 \\
\hline & 2 & 9 & 41,6 & 20,1 & 13,8 & 42,7 & 70,9 & \\
\hline \multirow[t]{2}{*}{$2 \mathrm{~cm}$} & 1 & 9 & 39,0 & 23,4 & 11,9 & 30,9 & 90,7 & 0,1 \\
\hline & 2 & 9 & 343,8 & 18,5 & 12,8 & 50,0 & 67,9 & \\
\hline \multirow[t]{2}{*}{$3 \mathrm{~cm}$} & 1 & 9 & 36,6 & 25,2 & 3,9 & 31,5 & 77,0 & 0,4 \\
\hline & 2 & 9 & 45,2 & 21,6 & 16,7 & 43,3 & 87,6 & \\
\hline \multirow[t]{2}{*}{$4 \mathrm{~cm}$} & 1 & 9 & 34,7 & 24,5 & 5,6 & 24,6 & 79,9 & 0,2 \\
\hline & 2 & 9 & 41,8 & 13,14 & 21,6 & 44,0 & 65,0 & \\
\hline \multirow[t]{2}{*}{$5 \mathrm{~cm}$} & 1 & 9 & 30,5 & 19,5 & 1,6 & 28,2 & 57,9 & 0,2 \\
\hline & 2 & 9 & 43,8 & 19,8 & 19,3 & 42,0 & 78,6 & \\
\hline
\end{tabular}

Grupo 1: pré operatório; Grupo 2: pós-operatório; $\mathbf{N}$ : número de pacientes analisados; DP: Desvio padrão. M-W: Mann-Whitney. 
Tabela 4 - Índice de relaxamento, segundo volume injetado, de pacientes submetidas a histerectomia.

\begin{tabular}{|c|c|c|c|c|c|c|c|c|}
\hline $\begin{array}{l}\text { Volume } \\
\text { injetado }\end{array}$ & Grupo & $\mathbf{N}$ & Média & DP & Mínima & Mediana & Máxima & $\begin{array}{c}\text { Análise } \\
\text { estatística } \\
\text { M-W }\end{array}$ \\
\hline \multirow[t]{2}{*}{$20 \mathrm{ml}$} & 1 & 9 & 37,7 & 36,9 & 2,7 & 28,6 & 111,8 & 0,8 \\
\hline & 2 & 9 & 36,0 & 18,8 & 0,0 & 39,6 & 39,6 & \\
\hline \multirow[t]{2}{*}{$40 \mathrm{ml}$} & 1 & 9 & 28,1 & 23,6 & 9,0 & 18,3 & 73,4 & 0,5 \\
\hline & 2 & 9 & 35,5 & 29,3 & 14,6 & 24,3 & 79,0 & \\
\hline \multirow[t]{2}{*}{$60 \mathrm{ml}$} & 1 & 9 & 27,0 & 20,5 & 7,0 & 20,1 & 67,6 & 0,2 \\
\hline & 2 & 9 & 41,0 & 13,3 & 17,6 & 44,4 & 49,9 & \\
\hline \multirow[t]{2}{*}{ Média } & 1 & 9 & 30,9 & 31,6 & 6,2 & 20,3 & 92,6 & 0,2 \\
\hline & 2 & 9 & 38,6 & 17,9 & 23,6 & 33,2 & 64,5 & \\
\hline
\end{tabular}

Grupo 1: pré operatório; Grupo 2: pós-operatório; $\mathbf{N}$ : número de pacientes analisados; DP: Desvio padrão. M-W: Mann-Whitney.

Tabela 5 - Pressões médias e capacidade de sustentação de contração mantida, segundo a altura do canal anal, de pacientes submetidas a histerectomia.

\begin{tabular}{|c|c|c|c|c|c|c|c|c|}
\hline $\begin{array}{l}\text { Contração } \\
\text { Mantida }\end{array}$ & Grupo & $\mathbf{N}$ & Média & DP & Mínima & Mediana & Máxima & $\begin{array}{c}\text { Análise } \\
\text { estatística } \\
\text { M-W }\end{array}$ \\
\hline Pressão & 1 & 9 & 104,7 & 29,1 & 42,9 & 110,1 & 149,0 & 0,06 \\
\hline Média & 2 & 9 & 75,6 & 30,8 & 38,2 & 71,0 & 132,0 & \\
\hline Cap. & 1 & 9 & 86,8 & 25,1 & 51,1 & 92,6 & 122,0 & 0,5 \\
\hline Sustentação & 2 & 9 & 89,6 & 34,5 & 37,4 & 98,4 & 144,0 & \\
\hline
\end{tabular}

Grupo 1: pré operatório; Grupo 2: pós-operatório; $N$ : número de pacientes analisados; DP: Desvio padrão. M-W: Mann-Whitney.

Para o diagnóstico da constipação intestinal temos uma variedade de exames, como os marcadores do trânsito colônico, manometria anal, eletromiografia, latência do nervo pudendo, ultrassonografia, sensibilidade retal e eletromiografia ${ }^{(11-18)}$, sendo a manometria anal a mais utilizada e divulgada, exame que optamos para realizar nas pacientes deste estudo.

A manometria anal tem sido motivo de muitas investigações e poderia contribuir para o diagnóstico do anismus ${ }^{(16)}$ e falhas esfincterianas e, mais recentemente , durante a realização do teste de sensibilidade retal que é realizado associado à manometria, tem contribuído com informações sobre a presença de hipersensibilidade visceral em alguns pacientes que apresentam dor abdominal relacionada à presença de fezes na ampola retal podendo sugerir a presença de síndrome do intestino irritável . ${ }^{(17)}$

As pacientes do nosso estudo não referiram constipação após a histerectomia, indo de encontro ao que ocorreu em outros estudos prospectivos relacionando constipação e histerectomia ${ }^{(2,9)}$. Além das pacientes não referirem constipação no pós-operatório, não houve diferença também quanto ao uso de laxativos, em concordância com outro estudo ${ }^{(7)}$. Estudos retrospectivos referem constipação após a histerectomia.

Em relação ao escore de constipação no pósoperatório podemos observar que ocorreu uma diferença significante no item presença de evacuação incompleta. Fica a pergunta de o porquê isso ter ocorrido. Poderia a ausência do útero ocasionar alterações anatômicas na pelve e o reto não se contrai de modo adequado? Alguma alteração na inervação provocada pela dissecção pélvica durante a cirurgia? Alguma dilatação retal com maior complacência e por isso o não esvaziamento retal completo? A histerectomia teria causado alguma alteração na dinâmica retal e anal e nos mecanismos que envolvem a evacuação? 
Tabela 6 - Escore de constipação de pacientes submetidas à histerectomia.

\begin{tabular}{llllrrrrc}
\hline \multicolumn{1}{c}{ Grupo } & N & Média & DP & Mínima & Mediana & $\begin{array}{c}\text { Máxima } \\
\text { Análise } \\
\text { estatística } \\
\text { M-W }\end{array}$ \\
\hline Frequencia & 1 & 9 & 0,1 & 0,3 & 0,0 & 0,0 & 1,0 & 0,9 \\
Intestinal & 2 & 9 & 0,2 & 0,6 & 0,0 & 0,0 & 2,0 & \\
Esforço & 1 & 9 & 0,7 & 0,4 & 0,0 & 1,0 & 1,0 & 0,09 \\
Evac. & 2 & 9 & 1,6 & 1,3 & 0,0 & 2,0 & 4,0 & \\
Evac. & 1 & 9 & 0,5 & 0,5 & 0,0 & 1,0 & 1,0 & 0,01 \\
Incompleta & 2 & 9 & 2,3 & 1,5 & 0,0 & 2,0 & 4,0 & \\
Dor & 1 & 9 & 0,6 & 0,5 & 0,0 & 1,0 & 1,0 & 0,3 \\
Abdominal & 2 & 9 & 1,2 & 1,3 & 0,0 & 1,0 & 4,0 & \\
Tempo (min) & 1 & 9 & 0,2 & 53,3 & 32,3 & 128,4 & 192,5 & 0,4 \\
& 2 & 9 & 1,4 & 61,8 & 18,1 & 89,8 & 215,2 & \\
Auxílio & 1 & 9 & 0,2 & 0,2 & 0,0 & 1,0 & 1,0 & 0,2 \\
Tentativa & 1 & 9 & 1,3 & 0,3 & 0,0 & 1,0 & 3,0 & \\
s/ sucesso & 2 & 9 & 0,2 & 0,4 & 0,0 & 0,0 & 1,0 & 0,1 \\
\hline
\end{tabular}

Grupo 1: pré operatório; Grupo 2: pós-operatório; N: número de pacientes analisados; DP: Desvio padrão. M-W: Mann-Whitney.

Também nos chamou a atenção que duas das nove pacientes apresentaram dor à distensão da ampola retal, sugerindo sinais de hipersensibilidade visceral, que pode ser um marcador de síndrome do intestino irritável ${ }^{(17)}$ sobre a dor abdominal em síndrome do intestino irritável. A presença espontânea de dor abdominal não foi diferente nos dois grupos, mas é um grupo pequeno e seria interessante um maior grupo de estudo. Os nossos achados sugerem a provável relação já descrita anteriormente sobre a existência da síndrome do intestino irritável no pós-operatório de histerectomia $^{(5)}$.

Em relação à manometria não encontramos diferenças em relação às pressões de repouso, mas aos cinco $\mathrm{cm}$ esta diferença de pressão retal foi próxima, sugerindo que possa existir uma tendência caso a amostra de estudo seja maior. Quando observadas pressões de contração e evacuação não observamos diferenças, semelhante aos resultados de outro estudo prospectivo ${ }^{(4)}$, mas quando observamos a contração mantida os valores foram menores no pós-operatório com diferença muito próximo da significância estatística. Sendo a contração mantida reflexo da atividade do esfíncter externo do ânus e do puborretal, fica a pergunta de o porquê a histerectomia interfere nesta atividade muscular. Seriam alterações anatômicas interferindo na sustentação pélvica? . Ainda na manometria anal não observamos diferenças no relaxamento esfincteriano interno nos diferentes volumes, contrariando os achados de outro estudo prospectivo em que o volume para desencadear o reflexo inibitório anal foi significativamente maior após a histerectomia ${ }^{(4)}$.

A presença de necessidade de esforço evacuatório apresenta tendência a ser significante, e a necessidade de maior tempo evacuatório foi referida pela maioria das pacientes no pós-operatório, mas não apresentou diferença significativa.

Os achados deste estudo sugerem que alterações intestinais ocorrem após a histerectomia e são principalmente relacionadas ao reto, em que observamos as diferenças de pressão retal e a necessidade de maior tempo evacuatório para estas pacientes, assim como a sensação de evacuação incompleta que foi estatisticamente maior, sugerindo alterações retais. Entretando o nosso grupo de estudo foi restrito, principalmente devido a não adesão dos pacientes à realização voluntária do exame de manometria anal. A continuidade do estudo nos traria maiores dados para real conhecimento dos distúrbios da evacuação associado à histerectomia. 


\section{CONCLUSÕES}

A avaliação manométrica anal das pacientes após histerectomia não mostrou diferença em relação à pressão de repouso, contração e evacuação e no reflexo inibitório anal. A alteração intestinal encontradas foi a sensação de evacuação incompleta, estatiscamente maior no pós-operatório.

\section{AGRADECIMENTOS}

Aos Doutores Deomir Germano Bassi e Xenofonte Mazzini pelas sugestões na confecção desta pesquisa e apoio para a realização da mesma nos serviços de ginecologia e obstetrícia e cirurgia.

\begin{abstract}
Hysterectomy is the most common gynecologic surgical procedure of great bearing being the majority of the indications due to being disease. Among the complications of this surgical procedure are constipation, dyspareunia, gas and feces incontinence, evacuatory urgency, fecal escapes and abdominal distension. Constipation has been frequently found after a hysterectomy, especially in retrospective studies. Objective: Study the incidence of constipation after hysterectomy and the manometrical alterations related to them. Methods: Prospective study in nine patients subjected to total abdominal hysterectomy due to myoma. A manometrical study and application of the constipation score adopted by "Sociedade Brasileira de Motilidade Digestiva" was performed in the pre and sixty days of post-operative. Results: The autors found presence of incomplete evacuation that did not occur in the pre-operative. Manometrical alterations were found and two of the patients presented abdominal pain to the rectal ampule distension in the tolerable maximum volume checking. Conclusions: The findings of this study suggest that intestinal alterations occur after hysterectomy and are specially related to the rectum.
\end{abstract}

Key words: Hysterectomy, constipation diagnosis, surgical procedures, operative, postoperative complications.

\section{REFERENCIAS}

1. Van Dam J H; Gosseelink M J; Drogendijk A C; Hop W C J; Schouten W R. Changes in bowel function after hysterectomy. Dis Colon Rectum 1997; 40: 1342-7.

2. Altman D; Zetterstrom J; López A; Pollack J; Nordenstam J; Mellgren A. Effect of hysterectomy on bowel function. Dis Colon Rectum 2004; 47(4): 502-9.

3. Forsgren C; Zetterstron J; López A; Nordenstam J; Anzen B; Altman D. Effects of hysterectomy on bowel function: a threeyear, prospective cohort study. Dis Colon Rectum 2007; 50: 1139-45.

4. Roossel A; Andersch B; Antov S; Berndtsson I; Oresland T; Hulten L. Does simple hysterectomy alter bowel function? Ann Chir Gynaecol 1997; 86:298-303.

5. Prior A; Stanley K M; Smith A R B; Read n w. Relation between hysterectomy and the irritable bowel: a prospective study. Gut 1992; 33: 814-17.

6. Heaton K W; Parker D; Cripps H: Bowel function and irritable bowel simptons after hysterectomy and cholecystectomy - a population based study. Gut 1993; 34:1108-11.

7. Taylor T; Smith A N; Fulton M. Effects of hysterectomy on bowel bladder function. Int J Colorectal Did 1990; 5: 22831.

8. Possover M; Schneider A. Slow-transit constipation after radical hysterectomy type III. Surg Endosc 2002; 16(5):84750 .
9. Roovers J P; Van Der Bom J G; Huub Van Der Vaart C. Hysterectomy does not cause constipation. Acta Obstet Gynecol Scand 2006; 51: 1068-73.

10. Dykes S; Smilgin-humphreys S; Bass C- Chronic idiopathic constipation. A psychological enquiry. Eur J Gastroenterol Hepatol 2001; 13(1): 39-44.

11. Cesar MAP; Ortiz JA; Faria FG; Salgado FC; Salan FO; Branco GAR; et AL. Reprodutibilidade da manometria anal em mulheres sem distúrbios evacuatórios. Rev Bras Coloproct 2004; 24(1): 33-37.

12. Vieira EP; Pupo Neto J; Lacombe DLP. Contribuição da manometria ano retal na avaliação da constipação intestinal crônica. Rev Bras Coloproct 2005; 25(0): 348-60.

13. Cesar MAP; Klug WA; Capelhuchnick P; Ortiz JA; Mantovani AP; Antunes CAB; et AL. Reprodutibilidade de laudos de uma mesma manometria segundo diferentes examinadores. Rev bras coloproct 2007; 27(1): 58-62.

14. Cesar MAP; Klug WA; Aguida HAC; Ortiz JA; Bin FC; Kapelhuchnik P. A presença de retocele interfere nos resultados de exames de fisiologia anal? Rev Bras Coloproct 2008; 28(3): 329-33.

15. Cesar MAP; Klug WA; Aguida HAC; Ortiz JA; Fang CB; Capelhuchnik P. Alterações das pressões anais em pacientes constipados por defecação obstruída. Rev Bras Coloproct 2008; 28(4): 402-08.

16. Cesar MAP; Klug WA; Ortiz JA; BIN FC; Kapelhuchnik P. Diagnóstico do anismus através dos exames de fisiologia anal. Rev Bras Coloproct 2009; 29(2): 192-96. 
17. Cesar MAP e Oliveira, CC: Existe importância na utilização da manometria anal no diagnóstico da sindrome do intestino irritável? Rev Bras Coloproct 2009; 29(3):358-362

18. Cesar MAP, Klug WA - Fisiologia Anorretal e cirurgia - Investigação dos distúrbios de evacuação (Constipação intestinal e incontinência fecal). In Speranzini MB, Deutsch CR, Yagi OK. Manual de diagnóstico e tratamento para o residente de cirurgia. 2009 (2): 1465-72

\section{Endereço para correspondência:} MARIA AUXILIADORA PROLUNGATTI CESAR

Serviço de Clínica Cirúrgica do hospital Universitário de Taubaté Avenida Granadeiro Guimarães, 270

Taubaté - SP

E mail: prolungatti@uol.com.br 